GLASNIK MATEMATIČKI

Vol. 39(59)(2004), $333-335$

\title{
A SELECTION THEOREM FOR SIMPLEX-VALUED MAPS
}

\author{
IVAN IVANŠIĆ AND LEONARD R. RUBIN \\ University of Zagreb, Croatia and University of Oklahoma, USA
}

\begin{abstract}
The purpose of this short note is to prove the following theorem. Let $X$ be a hereditarily normal paracompact Hausdorff space, $K$ be a simplicial complex, and $\sigma: X \rightarrow K$ be a function. Suppose that $\left\{U_{\alpha} \mid \alpha \in \Gamma\right\}$ and $\left\{f_{\alpha} \mid \alpha \in \Gamma\right\}$ are collections such that for each $\alpha \in \Gamma$, $f_{\alpha}$ is a map of $U_{\alpha}$ to $|K|$, and if $x \in U_{\alpha}$, then $f_{\alpha}(x) \in \sigma(x)$. Assume further that $\left\{U_{\alpha} \mid \alpha \in \Gamma\right\}$ is an open cover of $X$. Then there exists a map $f: X \rightarrow|K|$ such that for each $x \in X, f(x) \in \sigma(x)$.
\end{abstract}

\section{INTRODUCTION}

Recently ([2]) S. Mardešić gave a proof of the following lemma which had previously appeared in [1] with an incorrect proof. (We have added an additional conclusion (4), which follows from the proof of this lemma). The reader should recall that if $K$ is a simplicial complex and $f: A \rightarrow|K|$ and $g: A \rightarrow|K|$ are functions, then $f$ and $g$ are said to be contiguous if for each $x \in A$ there exists a simplex $\sigma \in K$ such that $f(x), g(x) \in \sigma$.

Lemma 1.1. Let $X$ be a normal space and $K$ a simplicial complex. Let $A \subset X$ be a closed set and $V, U \subset X$ be open sets such that $A \subset V \subset \bar{V} \subset U$. If $h: U \rightarrow|K|$ and $g: V \rightarrow|K|$ are maps such that $h \mid V$ and $g$ are contiguous, then there exists a map $k: U \rightarrow|K|$ such that:

(1) $k$ and $h$ are contiguous,

(2) $k|A=g| A$,

(3) $k|U \backslash V=h| U \backslash V$, and

(4) if $x \in V$ and $h(x), g(x) \in \sigma \in K$, then $k(x) \in \sigma$.

2000 Mathematics Subject Classification. 54C65, 54C05, 54E20.

Key words and phrases. Contiguous functions, continuous function, hereditarily paracompact, polyhedron, selection, simplex, simplicial complex, stratifiable space. 
This lemma along with its proof motivated us to think about a situation in which there might be a "piecewise" contiguous set of maps. In the next section we shall apply Lemma 1.1 along with the "local-to-global" technique of E. Michael to prove our main result, Theorem 1.2, a proposition about amalgamating a piecewise contiguous collection of maps into one map. This theorem applies to the class of hereditarily normal paracompact Hausdorff spaces and thus includes all metrizable spaces, and even the larger class of stratifiable spaces (information about stratifiable spaces can be found, e.g., in $[1])$.

If $K$ is a simplicial complex, $X$ is a space, $\sigma: X \rightarrow K$ is a function, and $\left\{U_{\alpha} \mid \alpha \in \Gamma\right\}$ is an open cover of $X$, then one says that a collection $\left\{f_{\alpha}: U_{\alpha} \rightarrow|K| \mid \alpha \in \Gamma\right\}$ of maps is a selection of $\sigma$ if $f_{\alpha}(x) \in \sigma(x)$ for each $x \in U_{\alpha}$.

Here is the main result of this paper.

TheOREM 1.2. Let $X$ be a hereditarily normal paracompact Hausdorff space, $K$ be a simplicial complex, and $\sigma: X \rightarrow K$ be a function. Suppose that $\left\{U_{\alpha} \mid \alpha \in \Gamma\right\}$ is an open cover of $X$ and $\left\{f_{\alpha}: U_{\alpha} \rightarrow|K| \mid \alpha \in \Gamma\right\}$ is a selection of $\sigma$. Then there exists a selection $f: X \rightarrow|K|$ of $\sigma$.

It is worth noting (for example, Lemma $4^{\prime}$ of [2]) that the map $f$ of this theorem has the property that $f \mid U_{\alpha} \simeq f_{\alpha}$ for each $\alpha \in \Gamma$.

\section{Proof of Theorem}

Lemma 2.1. Let $X$ be a normal space and $U, Q$ be open subsets of $X$ such that $\{U, Q\}$ covers $X$. Suppose that $K$ is a simplicial complex, and $\sigma: X \rightarrow K$ is a function. Let $\{h: U \rightarrow|K|, g: Q \rightarrow|K|\}$ be a selection of $\sigma$. Then there exists a selection $l: X \rightarrow|K|$ of $\sigma$.

Proof. Using the shrinking lemma, let $\left\{U_{1}, Q_{1}\right\}$ be an open cover of $X$ such that $\bar{U}_{1} \subset U$ and $\bar{Q}_{1} \subset Q$. Using shrinking again, choose a closed cover $\left\{D_{1}, E_{1}\right\}$ of $X$ such that $D_{1} \subset Q_{1}$ and $E_{1} \subset U_{1}$.

Define $A=D_{1} \cap E_{1}$ and $V=U_{1} \cap Q_{1}$. Then $A \subset V \subset \bar{V} \subset \bar{U}_{1} \cap \bar{Q}_{1} \subset U$. Apply Lemma 1.1 to $h: U \rightarrow|K|$ and $g|V: V \rightarrow| K \mid$ to get a map $k: U \rightarrow|K|$ satisfying (1)-(4) of that lemma. Define a map $l: X \rightarrow|K|$ by,

$$
l(x)= \begin{cases}g(x) & \text { if } x \in D_{1}, \\ k(x) & \text { if } x \in E_{1} .\end{cases}
$$

An application of (2) of Lemma 1.1 shows that $l$ is a well-defined map. Since $x \in D_{1}$ implies that $l(x)=g(x) \in \sigma(x)$, then we only have to consider $x \in E_{1} \backslash D_{1}$. Hence $l(x)=k(x)$. Note that it must be true that $x \in U$. There are two cases.

Case 1. $x \in U \backslash V$. By (3) of Lemma 1.1, $l(x)=h(x) \in \sigma(x)$.

Case 2. $x \in V \subset U$. Then $h(x), g(x) \in \sigma(x)$, so by (4) of Lemma 1.1, $l(x)=k(x) \in \sigma(x)$. 
Let us state Theorem 3.6(a) of E. Michael ([3]).

Proposition 2.2. Let $X$ be a paracompact Hausdorff space and $\mathcal{G}$ a collection of subsets of $X$. Suppose that the following are true:

(1) $\mathcal{G}$ contains an open cover of $X$,

(2) If $U \in \mathcal{G}$ and $W$ is open in $U$, then $W \in \mathcal{G}$,

(3) if $U, V$ are open elements of $\mathcal{G}$, then $U \cup V \in \mathcal{G}$, and

(4) if $\mathcal{K} \subset \mathcal{G}$ is a pairwise disjoint collection of open subsets of $X$, then $\cup \mathcal{K} \in \mathcal{G}$.

Then the entire space $X$ is in $\mathcal{G}$.

We now give our proof of Theorem 1.2.

Proof. Let $\mathcal{G}$ be the collection of all open subsets $G$ of $X$ such that there exists a selection $g: G \rightarrow|K|$ of $\sigma \mid G$. We merely need to show that $X \in \mathcal{G}$, and we shall apply Proposition 2.2 to do this. One sees from the hypothesis that (1), (2), and (4) of Proposition 2.2 hold true. Using the fact that $X$ is hereditarily normal, we get (3) from Lemma 2.1 .

ACKNowledgements.

The authors are very thankful to the referee for making suggestions that significantly improved the exposition in this paper.

\section{REFERENCES}

[1] S. Mardešić, Extension dimension of inverse limits, Glas. Mat. Ser. III 35(55) (2000), 339-354.

[2] S. Mardešić, Extension dimension of inverse limits. Correction of a proof, Glas. Mat. Ser. III 39 (2004), 335-337.

[3] E. Michael, Local properties of topological spaces, Duke Mat. J. 21 (1954), 163-171.

I. Ivanšić

Department of Mathematics

University of Zagreb

Unska 3, P.O. Box 148

10001 Zagreb

Croatia

E-mail: ivan.ivansic@fer.hr

L.R. Rubin

Department of Mathematics

University of Oklahoma

Norman, Oklahoma 73019

USA

E-mail: lrubin@ou.edu

Received: 10.12.2003.

Revised: 04.01.2003. \& 23.08.2004. 